\title{
Las Educadoras y Educadores Sociales ante la Sociedad red
}

\author{
Marcos Cabezas González a \\ Sonia Casillas Martín ${ }^{b}$
}

\section{Resumen}

Este artículo presenta los resultados de una investigación cuyo propósito es el de identificar el grado de competencia digital autopercibido por los futuros Educadores Sociales, centrándose en las dimensiones de conocimiento, manejo, actitud hacia las TIC y en la variable de género, como base fundamental para el desarrollo profesional de los mismos en la Sociedad red. La metodología utilizada se incluye dentro de las denominadas ex-post-facto, es de tipo cuantitativo y se utilizó la técnica del cuestionario online para la recogida de la información. Los análisis llevados a cabo fueron de tipo descriptivo, inferencial y correlacional. Con los resultados obtenidos, podemos concluir que las Educadoras y los Educadores Sociales no están preparados para educar en la Sociedad red.

Palabras clave: Sociedad red. Tecnologías de la Información y la Comunicación. Competencia digital. Educación Social.

\section{Introducción}

\section{La Educación Social es}

un derecho de la ciudadanía que se concreta en el reconocimiento de una profesión de carácter pedagógico, generadora de contextos educativos y acciones mediadoras y formativas, que son ámbito de competencia profesional del Educador Social, posibilitando: a) la incorporación del sujeto de la educación a la diversidad de las redes sociales, entendida como el desarrollo de la sociabilidad y la circulación social; b) la promoción cultural y social, entendida como apertura a nuevas posibilidades de la adquisición de bienes culturales, que amplíen la perspectivas educativas, laborales, de ocio

\footnotetext{
a Universidade de Salamanca, Salamanca, España

b Universidade de Salamanca, Salamanca, España 


\section{y participación social (ASOCIACIÓN ESTATAL DE EDUCACIÓN} SOCIAL, 2007, p. 12).

El perfil profesional del Educador Social es amplio, ya que puede trabajar en contextos muy diversos, por lo que su profesión es polivalente y está adscrita, principalmente, a ámbitos laborales relacionados con proyectos y servicios dirigidos a: infancia y juventud; atención a personas discapacitadas; servicios sociales de atención primaria; servicios sociales de atención a la tercera edad; dinamización comunitaria; inserción comunitaria; inserción laboral; servicios penitenciarios; violencia de género; etc. (FULLANA et al, 2008).

Los cambios económicos y sociales han transformado la base material de la sociedad, y el procesamiento y la transferencia de información se han convertido en los pilares fundamentales de la productividad y el poder en la denominada Sociedad red (CASTELLS, 2006). Los incesantes avances científicos y tecnológicos, así como la globalización económica y cultural, impulsan la actividad de la sociedad actual y originan un rápido desfase en el conocimiento, además de provocar continuas transformaciones en las estructuras culturales, económicas y sociales, exigiendo a la ciudadanía una constante adaptación (CENTENO MORENO; CUBO DELGADO, 2013).

En este contexto, las Tecnologías de la Información y la Comunicación (TIC) han supuesto un cambio tan radical que es necesario formar a los ciudadanos para vivir en este tipo de sociedad (HERNÁNDEZ PRADOS; VICENT; SANCHEZ ESTEBAN, 2014). Las TIC están presentes en todos los ámbitos de la sociedad (CALVO MARIN; OSPINA OSPINA, 2014) y el Educador Social como profesional del ámbito socioeducativo no puede permanecer ajeno a ellas. Deberá convertirse en un

agente que potencia y facilita la adaptación del individuo a la sociedad, favoreciendo su socialización, y es precisamente, en la actualidad, donde este profesional adquiere mayor relevancia, dado que las tecnologías influyen en las interacciones con los demás, en la incorporación activa como ciudadanos, en el desenvolvimiento en la sociedad, y en el propio desarrollo comunitario y social (SAMPEDRO REQUENA, 2015, p. 11).

El Educador Social puede encontrar en las TIC un potencial técnico para su formación continua, además de nuevos caminos y alternativas para la acción socioeducativa (LÓPEZ MENESES; ESTEBAN IBÁÑEZ, 2008). Y es obvio 
que, si estos profesionales quieren utilizar las TIC en su desarrollo profesional, deben de estar alfabetizados digitalmente.

Son varias las razones que justifican el uso de las TIC por parte de estos profesionales (LÓPEZ MENESES; ESTEBAN IBÁÑEZ, 2008): en primer lugar, la incorporación de los avances tecnológicos es, a día de hoy, un hecho de la vida diaria que puede traernos beneficios (desarrollo cultural, participación social, nuevos canales de comunicación, más medios formativos, etc.). En segundo lugar, los Educadores Sociales tienen el importante papel de ayudar a los ciudadanos a adaptarse a los acelerados y constantes cambios que se producen en el contexto social, para que estos, de una forma crítica, se conviertan en usuarios activos de los nuevos medios tecnológicos y no queden excluidos de los espacios significativos de la vida social. En tercer lugar, el Educador Social de este milenio debe integrar estos medios telemáticos que facilitan nuevas formas de presentar los contenidos y nuevos procesos para adquirirlos. En cuarto lugar, el profesional de la Educación Social debe ser un agente social que reflexione sobre las TIC, quiera y esté capacitado para formar a usuarios que puedan relacionarse, interactuar, ser críticos y sacar provecho de las mismas para la creación de su proyecto de vida futuro. En quinto lugar, las actuaciones socioeducativas deben de tener una visión más comunitaria y sistémica. Las opciones que pueden ofrecer las TIC en los proyectos sociales podrán convertirse en nuevas propuestas de actuación y en nuevas respuestas a las actuales necesidades socioeducativas desde los distintos ámbitos de la Educación Social. Y en sexto y último lugar, los Educadores Sociales deberán aprovechar las potencialidades de las TIC para integrarlas en la formación, la investigación y la intervención socioeducativas, para que de esta manera puedan servir como medio de desarrollo a nivel individual y como soporte de participación y emancipación social.

La formación tecnológica es una necesidad inevitable para cualquier Educador Social que quiera estar en armonía con su época. Debemos comenzar por conocer, investigar, experimentar e innovar con las TIC con actitud participativa y crítica ante la Sociedad red. La Educación Social debe reflexionar profundamente sobre el uso educativo de las mismas, sobre su adecuación a los diferentes ámbitos profesionales, sobre los impactos que provocan, sobre las nuevas didácticas y los efectos que tienen los nuevos entornos educativos virtuales en las relaciones educativas. Pero habrá que reflexionar, también, sobre los posibles riesgos y peligros que vienen de la mano de las TIC (PÉREZ, 2010).

"La competencia digital engloba el uso seguro y crítico de las tecnologías de la sociedad de la información (TSI) para el trabajo, el ocio y la comunicación" 
(UNIÓN EUROPEA, 2007, p. 7). Desde el punto de vista de un Educador Social, entendemos la competencia digital como el conjunto de conocimientos, procedimientos, habilidades, valores y actitudes sobre las TIC, que debe poseer para que, profesionalmente, sea capaz de: alfabetizar tecnológicamente a sus educandos, colaborar en la erradicación de la brecha digital, contribuir a que los ciudadanos no se queden al margen de la Sociedad red (e-exclusión), trabajar en aras de la e-inclusión, y contribuir al empoderamiento de las personas y los colectivos sociales por medio de las Tecnologías para el Empoderamiento y la Participación (TEP) (REIG, 2012).

Es necesario que la competencia digital sea una capacidad transversal en la formación inicial del Educador Social, ya que este debe conseguir "el éxito en un mundo complejo e interconectado que se enfrenta a un cambio rápido de tipo tecnológico, cultural, económico, informativo y demográfico" (KAMPYLIS; PUNIE; DEVINE, 2015, p. 7).

Existen diferentes investigaciones cuyo objeto de estudio son las TIC y la Educación Social (CASTAÑEDA QUINTERO; GUTIÉRREZ PORLÁN; ROMÁN GARCÍA, 2014; ECHALAR; PEIXOTO, 2017; PALENCIA JIMÉNEZ; JIMÉNEZ FERNÁNDEZ, 2016; MARTÍNEZ PÉREZ, 2015; MIRANDA; FABBRI; GUERRA, 2010; RIVAS REBAQUE; CISNEROS DE BRITTO; GÉRTRUDIX BARRIO, 2015). También estudios sobre la influencia de la variable de género en relación al conocimiento, manejo y actitud hacia las TIC (BARRANTES CASQUERO; CASAS GARCIA; LUENGO GONZÁLEZ, 2014; LAW; PELGRUM; PLOMP, 2008; ROBLIZO COLMENERO; CÓZAR GUTIÉRREZ, 2015; WAITE, 2004; YUEN; MA, 2002). En esta línea, nuestra investigación se centró en comprobar si los futuros Educadores Sociales tenían la competencia tecnológica necesaria para encarar su desarrollo profesional en la Sociedad red y si esta variaba de acuerdo al género, como ya había sido estudiado en otros campos cercanos al de la Educación Social.

\section{Método}

Se empleó una metodología ex-post-facto y el cuestionario online para la recogida de datos (BERENDS, 2006). En este tipo de trabajos no se manipulan directamente las variables y la única finalidad es la de establecer relaciones entre las mismas. Esta metodología es muy empleada en el ámbito de la investigación educativa y de las ciencias sociales, y trata de describir, analizar relaciones y estudiar las variables objeto de análisis. 
Por otro lado, al no existir manipulación de variables, tratar de evaluar la realidad existente y limitarnos a describir los fenómenos y datos, este trabajo es de carácter descriptivo (BISQUERRAALZINA, 2004). Por último, señalar su carácter correlacional (MCMILLAN; SCHUMACHER, 2005), al estudiar las relaciones entre distintas variables dependientes, además de la influencia del género, como variable independiente.

\subsection{Objetivos}

Intentamos confirmar si los futuros Educadores Sociales creen poseer la competencia tecnológica necesaria para encarar su desarrollo profesional en la Sociedad red. Para ello, se plantearon dos objetivos: (1) constatar su grado de competencia digital autopercibida y (2) verificar si la variable género influye en el mismo.

\subsection{Muestra}

Con una población objeto de estudio de 280 futuros profesionales de la Educación Social, se utilizó un tipo de muestreo aleatorio simple con un margen de error de $+1 \%$ para un nivel de confianza del $95 \%$, y la muestra final quedó compuesta por 273 sujetos, de los cuales, el 13,7\% eran hombres y el 86,3\% mujeres, con edades comprendidas entre los 17 y los 26 años, y mayoritariamente procedían de las Comunidades Autónomas de Castilla y León y Extremadura (España) (76,3\%).

La mayoría de los participantes poseían dispositivos tecnológicos con los que poder acceder al mundo digital. Casi todos ellos disponían de ordenador portátil (98,7\%), de Smartphone (100\%), y de cámara de fotos digital (87,2\%). Más de la mitad poseían ordenador de sobremesa $(60,3 \%)$, un Sistema de Posicionamiento Global o GPS (44,9\%) y Tablet (44,9\%). Son menos los que tenían dispositivo para la lectura de eBook $(23,1 \%)$ y cámara de video digital $(34,6 \%)$. Solo un $3,8 \%$ disfrutaban de algún tipo de wearable.

\subsection{Instrumento}

Se utilizó un cuestionario online de respuesta directa, pre-codificado y transversal, diseñado ad hoc para identificar el grado de competencia digital en estudiantes universitarios. Para asegurar la cumplimentación correcta e independiente por parte de los estudiantes, fue aplicado directamente por los investigadores.

Para responder se utilizó una escala ordinal tipo Likert de 0 a 10, siendo 0 el valor mínimo, 10 el máximo y 5 el punto medio que indica si el sujeto se considera apto para realizar las acciones propuestas; y la opción de respuesta cerrada dicotómica. Para su validación estadística se utilizó el modelo de Rasch de un parámetro, una vez 
aceptados los supuestos de independencia local y uni-dimensionalidad. El cuestionario inicial fue optimizado tras la aplicación de una prueba piloto y sometido a juicio de expertos para la validación del contenido. Su consistencia interna se midió por medio del cálculo de la validez y la fiabilidad, y se constató una elevada fiabilidad a través del estadístico $\alpha$ de Cronbach tanto para cada una de las dimensiones $(\alpha$ Conocimiento $=0,94, \alpha$ uso $=0,88$ y $\alpha$ actitud $=0,95)$ como para la escala completa $(\alpha$ $=0,947)$. Previamente a este trabajo, se realizó un análisis exploratorio inicial de los ítems además de los pertinentes análisis psicométricos. Ello nos permitió identificar las diferentes dimensiones estructurales del instrumento anteriormente mencionadas.

El instrumento consta de 92 ítems y está estructurado en cinco bloques: (1) IDENTIFICACIÓN (ID): Datos de identificación personal (ítems 1-8); (2) POSESIÓN (PS): Dispositivos TIC que tienen (ítems 43-52); (3) CONOCIMIENTO $(\mathrm{CN})$, dividido en dos sub-apartados: conocimiento sobre conceptos relacionados con las TIC (CN_01; ítems 8-36) y conocimiento de dispositivos TIC (CN_02; ítems 37-42); (4) MANEJO (MJ), dividido en tres subapartados: manejo de dispositivos TIC (MJ_01; ítems 53-60), manejo de herramientas TIC (MJ_02; ítems 61-69) y manejo de servicios TIC (MJ_03; ítems 70-84); (5) ACTITUD (AC): valoración sobre las TIC de acuerdo a su necesidad e importancia para el futuro profesional de la educación (ítems 85-92).

\subsection{Tratamiento de los datos}

Mediante un análisis exploratorio inicial se comprobaron los supuestos paramétricos de normalidad y se aplicó para la evaluación de ajustes las pruebas de Kolmogorov-Smirnov y de Homocedasticidad de Levene. A partir de ellas se optó por la utilización de la prueba no paramétrica de contraste de hipótesis U de Mann-Whitney, situando el umbral para el nivel de significancia en 0,05. Esto nos permitió verificar la existencia de diferencias significativas en función de la variable predictora de género.

Para medir el nivel de significatividad se utilizó el cálculo del tamaño del efecto (TE) a partir del estadístico r (CORDER; FOREMAN, 2009), calculado del valor del estadístico de contraste obtenido en la prueba de la U de Mann-Whitney. Con esto se pretendió estudiar la relación directa que puedan practicar estas variables sobre las diferencias inter-grupo. Además, se evaluó la significatividad de las relaciones entre los diferentes bloques del cuestionario mediante la utilización de la correlación bivariada en función de dicha variable (GWET, 2012).

El tratamiento de los datos se centró en un análisis de tipo descriptivo, por medio del cálculo de estadísticos sobre las capacidades tecnológicas que poseen los 
futuros Educadores Sociales, además de realizar otro de tipo inferencial a partir de la comparación de medias. Todo ello por medio del software estadístico Statistical Package for the Social Sciences (SPSS v.22).

\section{Resultados}

Presentamos los principales resultados organizados en dos apartados. Por un lado, los referidos a la competencia digital autopercibida, y por otro, la influencia de la variable género en la misma.

\subsection{Estudio descriptivo previo}

En este primer análisis se exponen los estadísticos descriptivos de cada una de las competencias que componen los bloques de conocimiento $(\mathrm{CN})$, manejo (MJ) y actitud (AC).

\subsubsection{Conocimiento sobre conceptos y dispositivos TIC (CN_01 y CN_02)}

Los parámetros referidos a las medias y a las desviaciones típicas son los siguientes (tabla 1):

Tabla 1. Estadísticos descriptivos (conocimiento conceptos)

\begin{tabular}{lcc}
\hline \multicolumn{1}{c}{ CONOCIMIENTO (CN) } & & \\
\hline Conceptos (CN_01) & $\overline{\mathbf{x}}$ & SD \\
\hline TIC & 5,30 & 2,22 \\
Tecnología para el aprendizaje y el Conocimiento (TAC) & 3,00 & 2,40 \\
Tecnologías para el Empoderamiento y la Participación (TEP) & 1,36 & 1,932 \\
Web 2.0 & 1,86 & 2,740 \\
Web 3.0 & 1,47 & 2,186 \\
Wikipedia & 9,25 & 1,321 \\
Blogosfera & 3,64 & 3,293 \\
Podcast & 2,93 & 3,14 \\
Marcador social & 1,93 & 2,58 \\
Sindicación & 1,40 & 2,20 \\
Mashup & 1,34 & 2,47 \\
\hline Objeto de aprendizaje (OA) & 3,78 & 2,770 \\
\hline
\end{tabular}


Tabla 1. Estadísticos descriptivos (conocimiento conceptos) Continuation

\begin{tabular}{lcc}
\hline E-learning & 2,12 & 3,095 \\
M-learning & 0,78 & 1,37 \\
B-learning & 0,73 & 1,387 \\
MOOC & 0,68 & 1,452 \\
Almacenamiento en la nube & 6,74 & 2,95 \\
Realidad Virtual (RV) & 5,22 & 3,097 \\
Realidad Aumentada (RA) & 3,51 & 3,180 \\
Copyleft & 1,59 & 2,613 \\
Licencia Creative Commons (CC) & 1,41 & 2,70 \\
Brecha digital & 2,75 & 3,209 \\
E-exclusión & 2,49 & 3,31 \\
E-inclusión & 2,27 & 3,074 \\
Alfabetización digital & 3,60 & 3,307 \\
Ciberactivismo & 3,52 & 3,30 \\
E-participación & 1,74 & 2,863 \\
Empoderamiento & 1,56 & 2,560 \\
\hline
\end{tabular}

Fuente: Elaboración propia (2017)

Las valoraciones presentan una desviación típica muy alta, lo que indica que existe muy poca homogeneidad en las respuestas, debido a la existencia de diferentes niveles en los futuros Educadores Sociales, quienes se suspenden desde el punto de vista conceptual ya que, en la mayoría de los casos, la autoevaluación se sitúa por debajo del cinco. De todos los conceptos propuestos, solo el $14,2 \%$ son conocidos por los estudiantes, correspondiéndose este porcentaje con las nociones de Wikipedia, almacenamiento en la nube, TIC y realidad virtual.

Respecto al conocimiento de dispositivos, todos dicen saber lo que es una Tablet, un Smartphone y un GPS. El 98,6\% manifiestan conocer lo que es un ebook y el 91,8\% lo que es una Pizarra Digital Interactiva (PDI). Solo el 30,1\% dicen saber lo que es un wearable.

\subsubsection{Autoevaluación sobre capacidades para el manejo de dispositivos, herramientas y servicios TIC (MJ_01, MJ_02, MJ_03)}

En la mayoría de capacidades para el manejo de dispositivos (tabla 2), se autoevalúan con puntuaciones superiores a 5 , excepto en la utilización de 
wearable, en la que se suspenden $(X=1,86)$. Los dispositivos que mejor dicen manejan son la cámara de fotos digital $(\mathrm{X}=6,86, \mathrm{SD}=1,939)$, el Smartphone $(\mathrm{X}=7,59 ; \mathrm{SD}=1,884)$ y la Tablet $(\mathrm{X}=6,97, \mathrm{SD}=2,055)$.

En cuanto a las herramientas (tabla 3), aseguran que son capaces de manejar de forma notable las redes sociales y las de comunicación. También se aprueban en las de búsqueda, en las de documentación (buscadores, publicaciones electrónicas, recursos didácticos, objetos de aprendizaje, etc.) y en las de ofimática. Por contra, se suspenden cuando se trata de manejar herramientas para la formación (plataformas e-learning. Moodle. etc.).

Tabla 2. Estadísticos descriptivos (manejo de dispositivos)

\begin{tabular}{lcccc}
\hline \multicolumn{5}{c}{ MANEJO (MJ) } \\
Dispositivos (MJ_01) & $\overline{\mathbf{x}}$ & SD & Mínimo & Máximo \\
\hline Ordenadores & 5,29 & 2,208 & 0 & 10 \\
Cámara fotos digital & 6,86 & 1,939 & 0 & 10 \\
Cámara vídeo digital & 5,37 & 2,348 & 0 & 9 \\
Smartphone & 7,59 & 1,884 & 1 & 10 \\
Tablet & 6,97 & 2,055 & 0 & 10 \\
Lector e-Book & 5,85 & 2,827 & 0 & 10 \\
GPS & 5,71 & 3,034 & 0 & 10 \\
Wearable & 1,86 & 2,755 & 0 & 10 \\
\hline
\end{tabular}

Fuente: Elaboración propia (2017)

Tabla 3. Estadísticos descriptivos (manejo de herramientas TIC)

\begin{tabular}{lcccc}
\hline \multicolumn{1}{c}{ MANEJO (MJ) } & & & \\
\hline Herramientas (MJ_02) & $\overline{\mathbf{x}}$ & SD & Mínimo & Máximo \\
\hline Ofimáticas & 5,14 & 2,605 & 0 & 10 \\
De edición & 4,84 & 2,718 & 0 & 10 \\
De búsqueda y documentación & 5,25 & 2,448 & 0 & 10 \\
De trabajo colaborativo & 4,63 & 2,741 & 0 & 9 \\
De organización del tiempo & 4,21 & 2,809 & 0 & 9 \\
De comunicación & 8,32 & 1,580 & 2 & 10 \\
Para la formación & 3,95 & 2,862 & 0 & 9 \\
De almacenamiento en la nube & 4,01 & 3,323 & 0 & 10 \\
Redes sociales & 8,42 & 1,787 & 3 & 10 \\
\hline
\end{tabular}

Fuente: Elaboración propia (2017) 
Respecto a los servicios (tabla 4) su uso es bastante bajo y las valoraciones son más dispersas, con distribuciones muy heterogéneas (desviaciones típicas, en muchos casos, superiores a 3). Se ven muy competentes para manejar WhatsApp y utilizan más Facebook que Twitter. No tienen ni blog ni sitio web propio, no utilizan el almacenamiento en la nube y no consultan ni publican en wikis, pero sí usan Internet para compartir fotos y vídeos, aunque no para publicar y compartir presentaciones, audios, y mucho menos, contenidos.

\subsubsection{Autoevaluación sobre la necesidad e importancia de las TIC para el futuro profesional de la educación (AC)}

La actitud hacia las TIC (tabla 5) es muy positiva (medias próximas o superiores a 8). Reconocen que son necesarias y útiles para su futuro profesional, para economizar esfuerzo en su futura profesión y que pueden ayudarles en su desarrollo profesional. Consideran que el uso de las mismas es un medio útil para seguir formándose (valoración con la media más alta), a pesar de que sus valoraciones manifiestan que no utilizan herramientas tecnológicas para su formación.

Tabla 4. Estadísticos descriptivos (manejo de servicios TIC)

\begin{tabular}{|c|c|c|c|c|}
\hline \multicolumn{5}{|c|}{ MANEJO (MJ) } \\
\hline Servicios (MJ_03) & $\overline{\mathbf{x}}$ & SD & Mínimo & Máximo \\
\hline Twitter & 6,27 & 3,690 & 0 & 10 \\
\hline Facebook & 7,58 & 3,144 & 0 & 10 \\
\hline WhatsApp & 9,64 & 0,823 & 7 & 10 \\
\hline Internet para publicar y compartir fotos & 6,89 & 3,385 & 0 & 10 \\
\hline Internet para publicar y compartir vídeos & 5,01 & 3,799 & 0 & 10 \\
\hline $\begin{array}{l}\text { Internet para publicar y compartir } \\
\text { presentaciones }\end{array}$ & 3,40 & 3,692 & 0 & 10 \\
\hline Internet para publicar y compartir audio & 3,36 & 3,653 & 0 & 10 \\
\hline Chat, comunicación, videoconferencia & 6,12 & 3,321 & 0 & 10 \\
\hline Tengo mi propio blog & 1,18 & 2,756 & 0 & 10 \\
\hline Tengo mi propio sitio web & 0,55 & 1,893 & 0 & 10 \\
\hline Consulto wikis & 3,25 & 3,519 & 0 & 10 \\
\hline Publico en wikis & 0,60 & 1,927 & 0 & 10 \\
\hline $\begin{array}{l}\text { Internet para agregar y almacenar } \\
\text { contenidos }\end{array}$ & 0,52 & 1,701 & 0 & 9 \\
\hline Almacenamiento en la nube & 3,56 & 3,678 & 0 & 10 \\
\hline
\end{tabular}

Fuente: Elaboración propia (2017) 


\subsection{Estudio Inferencial. Variable predictora: género}

Se utilizó la variable género para comprobar si esta influía en la competencia digital desde el punto de vista del conocimiento, manejo y actitud hacia las TIC. De los contrastes realizados, en la mayoría de los aspectos estudiados no resultó muy significativa.

Una vez comprobadas las condiciones paramétricas de homogeneidad y normalidad, se realizó un contraste de hipótesis, para confirmar si las diferencias de medias para grupos independientes percibidas resultaban o no significativas, y se empleó la prueba U de Mann-Whitney para verificar la relevancia de estas diferencias en función del género.

Las diferencias observadas, aunque no significativas, en la mayoría de los casos son a favor de los alumnos (tabla 6). Son estos, a diferencia de las alumnas,

Tabla 5. Estadísticos descriptivos (actitud hacia las TIC)

\begin{tabular}{lcccc}
\hline ACTITUD (AC) & $\overline{\mathbf{x}}$ & SD & Mínimo & Máximo \\
\hline $\begin{array}{l}\text { TIC necesarias/útiles para mi futuro } \\
\text { profesional }\end{array}$ & 8,19 & 1,861 & 1 & 10 \\
$\begin{array}{l}\text { TIC ayudarán economizar esfuerzo/ } \\
\text { trabajo académico }\end{array}$ & 8,00 & 1,972 & 2 & 10 \\
$\begin{array}{l}\text { TIC ayudarán economizar esfuerzo/ } \\
\text { trabajo profesión }\end{array}$ & 8,10 & 1,781 & 2 & 10 \\
$\begin{array}{l}\text { TIC medio útil para seguir formándome } \\
\begin{array}{l}\text { Necesario TIC habituales desarrollo } \\
\text { profesional }\end{array}\end{array}$ & 8,37 & 1,752 & 2 & 10 \\
\begin{tabular}{l} 
Educador profesional competente en TIC \\
\hline
\end{tabular} & 8,64 & 2,077 & 0 & 10 \\
\hline
\end{tabular}

Fuente: Elaboración propia (2017)

Tabla 6. Prueba $U$ de Mann-Whitney para muestras independientes

\begin{tabular}{lccccccc}
\hline Bloques & V. Predictora & $\overline{\mathbf{x}}$ & SD & $\mathbf{U}$ & $\mathbf{V}$ & $\mathbf{p}$ & T.E \\
\hline \multirow{2}{*}{ CN_01 } & Femenino & 2,7249 & 1,46284 & & & & \\
& Masculino & 3,9185 & 2,91190 & 354 & 409 & 0,53 & 0,05 \\
MJ & Femenino & 4,9129 & 1,19221 & & & & \\
& Masculino & 5,8409 & 1,91256 & 407 & 462 & 0,14 & 0,04 \\
AC & Femenino & 8,2037 & 1,48603 & & & & \\
& Masculino & 7,1000 & 2,75121 & 266 & 321 & 0,43 & 0,03 \\
\hline
\end{tabular}

Fuente: Elaboración propia (2017) 
los que consideran que tienen mayores conocimientos en conceptos sobre TIC $(p>0,05)$ y un mayor manejo de las mismas $(p>0,05)$. Por el contrario, las alumnas muestran una actitud más positiva, aunque no con demasiada significancia, hacia las tecnologías $(\mathrm{p}>0,05)$.

Respecto al bloque de manejo (tabla 7), se encontraron diferencias en función de la variable predictora, aunque tampoco fueron significativas, ni en la utilización de herramientas $(p>0,05)$, ni en el uso de dispositivos $(p>0,05)$ ni en el de servicios $(p>0,05)$. El género masculino dice manejar ligeramente mejor las herramientas, los dispositivos y los servicios TIC que el femenino.

En el caso de las actitudes (tabla 8), los datos confirman que las mujeres manifiestan mayor disposición y mejores actitudes ante las tecnologías que los hombres. De la misma manera, son ellas la que consideran que las TIC son más necesarias y útiles.

Los valores obtenidos en el tamaño del efecto (TE.) son muy bajos $(<0,1)$ lo que explica las escasas diferencias entre el género masculino y el femenino en cuanto a conocimiento, uso y actitud hacia las TIC.

Tabla 7. Prueba U de Mann-Whitney para muestras independientes

\begin{tabular}{|c|c|c|c|c|c|c|c|}
\hline Manejo & V. Predictora & $\overline{\mathbf{x}}$ & SD & $\mathbf{U}$ & $\mathbf{V}$ & $\mathbf{p}$ & T.E \\
\hline \multirow{2}{*}{ MJ_01 } & Femenino & 5,5556 & 1,57519 & \multirow{2}{*}{420} & \multirow{2}{*}{475} & \multirow{2}{*}{0,92} & \multirow{2}{*}{0,05} \\
\hline & Masculino & 6,5250 & 1,82460 & & & & \\
\hline \multirow{2}{*}{ MJ_02 } & Femenino & 5,3016 & 1,57469 & \multirow{2}{*}{390} & \multirow{2}{*}{445} & \multirow{2}{*}{0,22} & \multirow{2}{*}{0,05} \\
\hline & Masculino & 6,1444 & 2,83668 & & & & \\
\hline \multirow{2}{*}{ MJ_03 } & Femenino & 3,8815 & 1,39304 & \multirow{2}{*}{408,5} & \multirow{2}{*}{463} & \multirow{2}{*}{0,13} & \multirow{2}{*}{0,03} \\
\hline & Masculino & 4,8533 & 1,58225 & & & & \\
\hline
\end{tabular}

Fuente: Elaboración propia (2017)

Tabla 8. Prueba U de Mann-Whitney para muestras independientes

\begin{tabular}{cccccccc}
\hline & V. Predictora & $\overline{\mathbf{x}}$ & SD & U & V & p & T.E \\
\hline \multirow{2}{*}{ AC } & Femenino & 8,20 & 1,48 & & & & \\
& Masculino & 7,10 & 2,75 & & 321 & 0,43 & 0,03 \\
\hline
\end{tabular}

Fuente: Elaboración propia (2017) 


\subsection{Estudio Correlacional}

La significatividad de las relaciones entre los distintos bloques del cuestionario fue evaluada mediante el Chi-Cuadrado de Pearson. El ajuste global ofrece valores del estadístico de contraste que indican la existencia del mismo $(\mathrm{p}<0,01)$, si tenemos en cuenta la sensibilidad de este tipo de contrastes cuando los tamaños de muestra son elevados (BACKHOFF ESCUDERO;GONZÁLEZ-MONTESINOS MARTÍNEZ, 2012).

Los bloques estudiados se correlacionan en función del género (tabla 9), dos relaciones a nivel 0,01 y una de ellas a nivel 0,05 . A mayor conocimiento de conceptos tecnológicos mejor manejo de la tecnología $\left(\mathrm{X}^{2}=0,683 ; \mathrm{p}<0,01\right)$, a favor de los hombres. También, con un grado de significatividad de 0,01 , tiene relación el manejo con la actitud, ya que a mejor manejo de la tecnología se manifestó una actitud más positiva hacia la misma, en este caso favoreciendo igualmente a los hombres $\left(X^{2}=0,295 ; p=0,01\right)$. La relación entre conocimiento y actitud es de densidad más débil (a nivel de 0,05 ) y en este caso son las mujeres las favorecidas por esta relación $\left(X^{2}=0,246 ; p>0,01\right)$.

Los tres sub-apartados referidos al manejo (tabla 10) se relacionan significativamente en función de la variable predictora, a favor del género masculino. Aquellos que mejor creen manejar los dispositivos tecnológicos, son los que mejor creen manejar tanto las herramientas $\left(X^{2}=0,729 ; p<0,01\right)$ como los servicios $\left(X^{2}=0,324\right.$; $\mathrm{p}<0,01)$. También se encontró una relación altamente significativa entre el manejo de herramientas y de servicios $\left(X^{2}=0,374 ; p<0,01\right)$.

Centrándose en las relaciones individuales planteadas en ambos modelos, los datos muestran valores superiores en el modelo masculino. Se puede observar,

Tabla 9. Correlación por bloques en función de la variable género

\begin{tabular}{lcccccc}
\hline $\begin{array}{l}\text { Correlación } \\
\text { bloques }\end{array}$ & V. Predictora & $\overline{\mathbf{x}}$ & SD & $\mathbf{X}^{\mathbf{2}}$ & $\mathbf{p}$ & $\mathbf{g l}$ \\
\hline CN_01-MJ & Mujeres & 2,7249 & 1,46284 & 0,683 & $0,000^{\star *}$ & 70 \\
& Hombres & 3,9185 & 2,91190 & & & \\
MJ-AC & Mujeres & 4,9129 & 1,19221 & & & 70 \\
& Hombres & 5,8409 & 1,91256 & 0,295 & $0,010^{\star *}$ & 70 \\
CN-AC & Mujeres & 8,2037 & 1,48603 & & & \\
& Hombres & 7,1000 & 2,75121 & 0,246 & $0,037^{*}$ & 70 \\
\hline
\end{tabular}

**Nivel de significatividad 0.01. * Nivel de significatividad 0.05 .

Fuente: Elaboración propia (2017) 
Tabla 10. Correlación en función de la variable género

\begin{tabular}{lcccccc}
\hline $\begin{array}{l}\text { Correlación } \\
\text { Manejo }\end{array}$ & V. Predictora & $\overline{\mathbf{x}}$ & SD & $\mathbf{X}^{\mathbf{2}}$ & $\mathbf{p}$ & $\mathbf{g l}$ \\
\hline MJ_01-MJ_02 & Femenino & 5,5556 & 1,57519 & 0,729 & 0,000 & 70 \\
& Masculino & 6,5250 & 1,82460 & & & \\
MJ_01-MJ_03 & Femenino & 5,3016 & 1,57469 & & & 70 \\
& Masculino & 6,1444 & 2,83668 & 0,324 & 0,005 & \\
MJ_02-MJ_03 & Femenino & 3,8815 & 1,39304 & 0,374 & 0,001 & 70 \\
\hline
\end{tabular}

** Nivel de significatividad 0.01

Fuente: Elaboración propia (2017)

no obstante, una tendencia favorable a las mujeres en efecto de la competencia conceptual sobre la actitudinal.

\section{Conclusiones}

En este trabajo se ha identificado el grado de competencia digital autopercibido por los futuros Educadores Sociales, centrándose en las dimensiones de conocimiento, manejo, actitud hacia las TIC y la variable de género, como base fundamental para el desarrollo profesional de los mismos en la Sociedad red.

Podemos concluir que los futuros profesionales de la Educación Social piensan que no están preparados para educar en la denominada Sociedad red y por ello es indispensable trabajar el desarrollo de la competencia digital en su formación inicial, porque las TIC además de estimular la creatividad y la innovación, contribuyen al diálogo intercultural, y juegan un importante papel en la superación de dificultades de aprendizaje individuales y colectivos (RODRÍGUEZ PÉREZ, 2015).

Los sujetos investigados no responden a los perfiles que tratan de caracterizar a una generación de aprendices nacida a partir de los años 80 (LENHART, 2001; PRENSKY, 2001; TAPSCOTT, 1998 y 2009; WHITE; LE CORNU, 2011). Nuestros resultados se identifican más con aquellas investigaciones que ponen de manifiesto la discutible existencia de una generación dotada especialmente para el conocimiento y el manejo de la tecnología (BENNETT; MATON, 2010; BULLEN; MORGAN; QAYYUM, 2011; CORRIN; LOCKYER; BENNETT, 2010; HELSPER; EYNON, 2010; LI; RANIERI, 2010; SELWYN, 2009), porque la muestra estudiada, aunque formada por personas nacidas a 
partir de los 80, revela no tener el nivel de alfabetización digital necesario para desarrollar un uso productivo de las TIC y lograr su mayor potencial en la Sociedad red (CASTAÑEDA QUINTERO; GUTIÉRREZ PORLÁN; ROMÁN GARCÍA, 2014), y no comienza su formación inicial con un nivel óptimo de competencia digital. Se podría decir que, más bien, forman parte de una generación nacida en la era digital acostumbrada a convivir a diario con la tecnología. Es cierto que muchos de ellos tienen más familiaridad y habilidades con la misma, pero por lo general, la utilizan principalmente en el ámbito del hogar y en actividades o tareas realizadas en los tiempos desocupados, libres y de ocio (GÓMEZ VARGAS, 2015; MUROS RUIZ; ARAGÓN CARRETERO; BUSTOS JIMÉNEZ, 2013).

La mayoría de Educadores Sociales sí encajan en el perfil de usuario multidispositivo de la Sociedad red, porque poseen más de un dispositivo TIC.

Si tenemos en cuenta las principales dimensiones estudiadas, se suspenden en el conocimiento de conceptos relacionados con las TIC, aunque manifiestan un conocimiento de dispositivos notable; se aprueban en su manejo y tienen una actitud muy positiva hacia las mismas. Resultados similares podemos encontrar en otras investigaciones (AUSÍN VILLAVERDE; DELGADO BENITO, 2015; CENTENO MORENO; CUBO DELGADO, 2013; HERNÁNDEZ PRADOS; LÓPEZ VICENT; SANCHEZ ESTEBAN, 2014).

Si tenemos en cuenta la variable predictora de género, los resultados obtenidos muestran, en la línea de otros estudios (CUADRADO GORDILLO; FERNÁNDEZ ANTELO; RAMOS SÁNCHEZ, 2009; LAW; PELGRUM; PLOMP, 2008; ROBLIZO COLMENERO; CÓZAR GUTIÉRREZ, 2015), la existencia de diferencias, aunque estas no son muy significativas, pudiendo concluir que existen escasas desigualdades entre la autopercepción del género masculino y del femenino en cuanto a conocimiento, uso y actitud hacia las TIC. Aun así, al igual que en otros trabajos (BARRANTES CASQUERO; CASAS GARCIA; LUENGO GONZÁLEZ, 2014; WAITE, 2004; YUEN; MA, 2002), encontramos diferencias en diversos apartados, en la mayoría de los casos a favor de los Educadores Sociales. Son ellos los que aseguran tener más conocimiento y manejo de las TIC, en contraposición con investigaciones como la de Cózar Gutiérrez y Roblizo Colmenero (2014) en las que son ellas. Por otro lado, son las Educadoras Sociales las que mejor actitud manifiestan, lo que nos puede indicar que tener mejor actitud o disposición no implica la adquisición de mayores conocimientos ni tan siquiera un mejor manejo de las diferentes tecnologías. 
Por último, si vinculamos el género con las relaciones entre las diferentes dimensiones estudiadas, podemos inferir que los hombres que mejor se valoran en el plano conceptual mejor lo hacen en el manejo tecnológico y a mejor manejo, mejor actitud hacia las TIC.

Para terminar, señalar que las limitaciones del trabajo se encuentran fundamentalmente en la composición de la muestra respecto al género (mayoritariamente femenino). Para futuros estudios sería aconsejable trabajar con muestras más paritarias desde el punto de vista del género. 


\title{
As Educadoras e os Educadores Sociais perante a Sociedade em rede
}

\section{Resumo}

Este artigo apresenta os resultados de uma investigação cuja intencão é identificar o grau de competência digital autopercebida pelos futuros Educadores Sociais, centrando-se nas dimensões de conhecimento, manejo, atitude para as TIC e na variável de gênero, como base fundamental para o desenvolvimento profissional deles e delas na Sociedade em rede. A metodologia empregada inclui-se dentro das denominadas ex-post-facto, é do tipo quantitativo e utilizou-se a técnica do questionário on-line para a coleta da informação. As análises levadas a cabo foram do tipo descritivo, inferêncial e correlacional. Com os resultados obtidos, podemos concluir que as Educadoras e os Educadores Sociais não estão preparados para educar na Sociedade em rede.

Palavras chave: Sociedade em rede. Tecnologias da Informação e Comunicação. Competência Digital. Educação Social.

\section{Social Educators and the Networked Society}

\begin{abstract}
This article presents the results of a research study aimed at identifying the degree of self-perceived digital competence of future Social Educators, focusing on the dimensions of their knowledge, use, and attitudes towards ICTs, and on the gender variable, considered a fundamental basis for their professional development in a networked society. The methodology used was quantitative ex-post-facto, with an online questionnaire to collect the data. Descriptive, inferential and correlational analyses were carried out. Results show that neither male nor female Social Educators are prepared to teach in a networked society.

Keywords: Networked Society. Information and Communication Technologies. Digital Competence. Social Education.
\end{abstract}




\section{Referencias}

ASOCIACIÓN ESTATAL DE EDUCACIÓN SOCIAL - Asedes. Documentos profesionalizadores. Barcelona, 2007. Disponible en: <http://www.eduso.net/ archivo/docdow.php?id=143>. Acceso en: 9 sep. 2017.

AUSÍN VILLAVERDE, V.; DELGADO BENITO, V. Aprendizaje percibido y actitud hacia las TIC desde la perspectiva de los PLE. Opción, v. 31, n. esp. 5, p. 91-110, 2015.

BACKHOFF ESCUDERO, E.; GONZÁLEZ-MONTESINOS MARTÍNEZ, M. J.. Evidencias de validez del cuestionario para docentes del estudio internacional sobre enseñanza y aprendizaje (TALIS 2009). Bordón, Revista de Pedagogia, v. 64, n. 2, p. 173-94, 2012.

BARRANTES CASQUERO, G.; CASAS GARCIA, L. M.; LUENGO GONZÁLEZ, R. F. Competencias tecnológicas de los profesores de Infantil y primaria de extremadura en función del género. Revista Iberoamericana de Informática Educativa, n. 19, p. 33-48, ene./jun. 2014.

BENNETT, S.; MATÓN, K. Beyond the 'digital natives' debate: towards a more nuanced understanding of students' technology experiences. Journal of Computer Assisted Learning, v. 26, n. 5, p. 321-31, oct. 2010. https://doi.org/10.1111/j.1365-2729.2010.00360.x

BERENDS, M. Survey methods in educational research. In: GREEN, J.; CAMILI, G.; ELMORE, P. B. (Eds.). Handbook of complementary methods in education research. Washington, DC: American Educational Research Association, 2006. p. 623-41.

BISQUERRA ALZINA, R. (Coord.). Metodología de la investigación educativa. Madrid: La Muralla, 2004.

BULLEN, M.; MORGAN, T.; QAYYUM. A. Digital learners in higher education: generation is not the issue. Canadian Journal of Learning and Technology, v. 37, n. 1, p. 1-24, 2011. https://doi.org/10.21432/T2NC7B

CALVO MARIN, D. C.; OSPINA OSPINA, D. H. Jóvenes y TIC: una mirada desde la vida cotidiana. Textos y sentidos, n. 9, p. 87-105, ene./jun. 2014.

CASTAÑEDA QUINTERO, L.; GUTIÉRREZ PORLÁN, I.; ROMÁN GARCÍA, M. M. Enriqueciendo la realidad: realidad aumentada con estudiantes de educación social. @Tic, Revista d'Innovació Educativa, n. 12, p. 15-25, ene./jun. 2014. https://doi.org/10.7203/attic.12.3544 
CASTELLS, M. La Sociedad Red: una visión global. Madrid: Alianza, 2006.

CENTENO MORENO, G.; CUBO DELGADO, S. Evaluación de la competencia digital y las actitudes hacia las TIC del alumnado universitario. Revista de Investigación Educativa, v. 31, n. 2, p. 517-36, 2013. https://doi. org/10.6018/rie.31.2.169271

CORDER, G.; FOREMAN, D. Nonparametric statistics for non-statisticians: a step-by-step approach. Hoboken, NJ: John Wiley \& Sons, 2009.

CORRIN, L.; LOCKYER, L.; BENNETT, S. Technological diversity: an investigation of students' technology use in everyday life and academic study. Learning, Media and Technology, v. 35, n. 4, p. 387-401, 2010. https://doi.org/ 10.1080/17439884.2010.531024

CÓZAR GUTIÉRREZ, R.; ROBLIZO COLMENERO, M. La competencia digital en la formación de los futuros maestros: percepciones de los alumnos de los grados de maestro de la facultad de educación de Albacete. RELATEC, Revista Latinoamericana de Tecnología Educativa, v. 13, n. 2, p. 119-33, dic. 2014. https://doi.org/10.17398/1695-288X.13.2.119

\section{CUADRADO GORDILLO, I.; FERNÁNDEZ ANTELO, I.; RAMOS}

SÁNCHEZ, J. L. Impacto de las TICs en las actitudes, formación y utilización del profesorado en el contexto escolar extremeño: estudios realizados entre 1998 y 2006. Campo Abierto, Revista de Educación, v. 28, n. 1, p. 13-33, 2009.

ECHALAR, A. D. L. F.; PEIXOTO, J. Programa um computador por aluno: o acesso às tecnologias digitais como estratégia para a redução das desigualdades sociais. Ensaio: Avaliação e Políticas Públicas em Educação, Rio de Janeiro, v. 25, n. 95, p. 393-413, abr./jun. 2017. https://doi.org/10.1590/ s0104-40362017002501155

FULLANA, J. et al. La inserción laboral de los diplomados en educación social: análisis de las características de los procesos de inserción y reconcomiendo profesional. Bordón, Revista de Pedagogia, n. 59, n. 4, p. 565-80, 2008.

GÓMEZ VARGAS, H. Jóvenes y la vida digital: de hacer vida (pos) moderna. Entretextos, v. 7, n. 19, p. 1-9, abr./jul. 2015.

GWET, K. Handbook of inter-rater reliability: the definitive guide to measuring the extent of agreement among raters. Piedmont, CA: Advanced Analytics, 2012. 
HERNÁNDEZ PRADOS, M. A.; VICENT, P L..; SANCHEZ ESTEBAN, S. La comunicación en la familia a través de las TIC, percepción de los adolescentes. Pulso: Revista de Educación, , n. 37, p. 35-58, 2014.

HELSPER, E. J.; EYNON, R. Digital natives: where is the evidence? British Educational Research Journal, v. 36, n. 3, p. 503-20, 2010. https://doi. org/10.1080/01411920902989227

KAMPYLIS, P.; PUNIE, Y.; DEVINE, J. Promoción de un aprendizaje eficaz en la era digital: un marcos europeo para organizaciones educativas digitalmente competentes. Madri: Joint Research Centre, 2015. (Ciencia para la política, informe del JRC).

LAW, N.; PELGRUM, W.J.; PLOMP, T. (Eds.). Pedagogy and ICT use in schools around the world: findings from the IEA sites 2006 study. New York, NY: Springer, 2008. (CERC studies in comparative education).

LENHART, A. Teenage life online: the rise of the instant message generation and the internet's impact on friendship and family relations. Washington, DC: Pew Internet y American Life Project, 2001.

LI, Y.; RANIERI, M. Are 'digital natives' really digitally competent? a study on chinese teenagers. British Journal of Educational Technology, v. 41, n. 6, p. 1029-42, 2010. https://doi.org/10.1111/j.14678535.2009.01053.x

LÓPEZ MENESES, E.; ESTEBAN IBÁÑEZ, M. La educación social y las nuevas tecnologías de la información y la comunicación: nuevos espacios en la construcción e intervención socioeducativa. Revista Latinoamericana de Estudios Educativos, v. 38, n. 1-2, p. 255-87, 2008.

MARTÍNEZ PÉREZ, A. Uso y percepción de las TIC por educadores y educadoras sociales en su tarea profesional. RES, Revista de Educación Social, n. 20, p. 1-11, ene. 2015.

MCMILLAN, J.; SCHUMACHER, S. Investigación educativa: una introducción conceptual. 5. ed. Madrid: Pearson, 2005.

MIRANDA, M. J.; FABBRI, M.; GUERRA, L. Recursos tecnológicos y enseñanza-aprendizaje de competencias en educación social: una experiencia internacional de constructivismo social en el EEES. Educación Social, n. 44, p. 55-73, 2010. 
MUROS RUIZ, B.; ARAGÓN CARRETERO, Y.; BUSTOS JIMÉNEZ, A. La ocupación del tiempo libre de jóvenes en el uso de videojuegos y redes. Comunicar, v. 20, n. 40, p. 31-9, 2013. https://doi.org/10.3916/C40-2013-02-03

PALENCIA JIMÉNEZ, R. G.; JIMÉNEZ FERNÁNDEZ, C. F. La brecha de género en la educación tecnológica. Ensaio: Avaliação e Políticas Públicas em Educação, Rio de Janeiro, v. 24, n. 92, p. 743-71, jun./sep. 2016. https://doi.org/10.1590/S0104-403620160003000010

PÉREZ, J. A. Educación social y tecnologías de la información y la comunicación: impactos y retos, reflexiones de un educador-TIC. RES, Revista de Educación Social, n. 11, 2010.

PRENSKY, M. Digital natives, digital inmigrants part 1. On the Horizon, v. 9, n. 5, p. 1-6, 2001. https://doi.org/10.1108/10748120110424816

REIG, D. Socionomía: ¿vas a perderte la revolución social? Barcelona: Deusto, 2012.

RIVAS REBAQUE, B.; CISNEROS DE BRITTO, J. C.; GÉRTRUDIX BARRIO, F. Análisis acerca de las claves en las políticas educativas para el empoderamiento ciudadano. EDUTEC-e, Revista Electrónica de Tecnología Educativa, n. 53, p. 1-19, sep. 2015. https://doi.org/10.21556/edutec.2015.53.300

ROBLIZO COLMENERO, M.; CÓZAR GUTIÉRREZ, R. Usos y competencias en TIC en los futuros maestros de educación infantil y primaria: hacia una alfabetización tecnológica real para docentes. Pixel-Bit, Revista de Medios y Educación, n. 47, p. 23-39, jul. 2015. https://doi.org/10.12795/pixelbit.2015.i47.02

RODRÍGUEZ PÉREZ, I. La importancia de las competencias digitales de los docentes, en la sociedad del conocimiento. Revista Iberoamericana de Producción Académica y Gestión Educativa, n. 2, p. 1-12, ene./jul. 2015.

SAMPEDRO REQUENA, B. E. Las TIC y la educación social en el siglo XXI. Edmetic, Revista de Educación Mediática y TIC, v. 5, n. 1, p. 8-24, 2015. https://doi.org/10.21071/edmetic.v5i1.4014

SELWYN, N. The digital native: myth and reality. Aslib, Journal of Information Management, v. 61, n. 4, p. 364-79, 2009. https://doi.org/10.1108/00012530910973776

TAPSCOTT, D. Growing up digital: the rise of the net generation. New York, NY: McGraw-Hill, 1998. 
TAPSCOTT. Grown up digital: how the net generation is changing your world. New York, NY: McGraw-Hill, 2009.

UNIÓN EUROPEA. Comisión Europea. Competencias clave para el aprendizaje permanente: un marco de referencia europeo. Luxemburgo: Oficina de Publicaciones Oficiales de las Comunidades Europeas, 2007.

WAITE, S. Tools for the job: a report of two surveys of information and communications technology training and use for literacy in primary schools in the West of England. Journal of Computer Assisted Learning, n. 20, n. 1, p. 11-20, feb. 2004.

WHITE, D. S.; LE CORNU, A. Visitors and residents: a new typology for online engagement. First Monday, The Peer Reviewed Journal on the Internet, v. 16 , n. 9, sep. 2011.

YUEN, A. H. K.; MA, W. W. K. Gender differences in teacher computer acceptance. Journal of Technology and Teacher Education, v. 10, n. 3, p. 365-82, 2002.

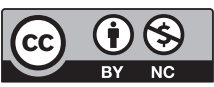

\section{Informações dos autores}

Marcos Cabezas González: Doctor en Pedagogía por la Universidad Pontificia de Salamanca. Profesor en el Departamento de Didáctica, Organización y Métodos de Investigación de la Universidad de Salamanca (España). Profesor en el Máster Tecnologías de la Información y la Comunicación en Educación: Análisis y diseño de procesos, recursos y prácticas formativas, de la Universidad de Salamanca (España). Investigador del Grupo de Investigación-Innovación en Tecnología Educativa de la Universidad de Salamanca (GITE-USAL). Contacto: mcabezasgo@usal.es

iD https://orcid.org/0000-0002-3743-5839

Sonia Casillas Martín: Doctora en Pedagogía por la Universidad Pontificia de Salamanca. Profesora en el Departamento de Didáctica, Organización y Métodos de Investigación de la Universidad de Salamanca (España). Profesora en el Máster Tecnologías de la Información y la Comunicación en Educación: Análisis y diseño de procesos, recursos y prácticas formativas, de la Universidad de Salamanca (España). Investigadora del Grupo de Investigación-Innovación en Tecnología Educativa de la Universidad de Salamanca (GITE-USAL). Contacto: scasillasma@usal.es

iD https://orcid.org/0000-0001-5304-534X 